\title{
Evaluación de la contaminación físico-química y microbiológica de aguas del estero salado
}

\section{Evaluation of the physical-chemical and microbiological contamination of salty estuary waters}

\section{Avaliação da contaminação físico-química e microbiológica das águas salgadas dos estuários}

Jesús E. Rodríguez-Villacis ${ }^{\mathrm{I}}$

jesusrodriguez@uees.edu.ec

Alberto Hernández-Gavilánez II

Carlos Silva-Huilcapi III

carlos.silvah@ug.edu.ec

Haydee M. Alvarado-Alvarado IV

haydee.alvaradoa@ug.edu.ec

Raisa S. Mariscal-García V

raisa.mariscal@hotmail.com

Frella S. García-Larreta VI

soraya.garcial@ug.edu.ec

Walter E. Mariscal-Santi VII

walter.mariscals@ug.edu.ec

Recibido: 30 de enero de 2017 * Corregido: 20 de febrero de 2017 * Aceptado: 20 junio de 2017

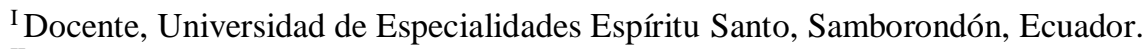

${ }^{\mathrm{II}}$ Docente, Universidad de Guayaquil, Guayaquil. Ecuador.

${ }^{\text {III }}$ Docente, Universidad de Guayaquil, Guayaquil. Ecuador.

${ }^{\text {IV }}$ Docente, Universidad de Guayaquil, Guayaquil. Ecuador.

${ }^{\vee}$ Docente, Universidad de Guayaquil, Guayaquil. Ecuador.

${ }^{\mathrm{VI}}$ Docente, Universidad de Guayaquil, Guayaquil. Ecuador.

${ }^{\mathrm{VII}}$ Docente, Universidad de Guayaquil, Guayaquil. Ecuador.
} 


\section{Resumen}

El presente trabajo de investigación comprende de un estudio concreto de evaluación de los niveles de contaminación de las aguas del Estero Salado ubicados en el puente Gómez Rendón, cantón Guayaquil, provincia del Guayas, cuyo objetivo fue determinar la contaminación físico-química y microbiológica de las aguas del Estero Salado, sector Sur-Oeste, de la ciudad de GuayaquilEcuador, utilizando procedimientos instrumentales de laboratorios emprendiendo acciones de educación y prevención ambiental. Para llevar a cabo nuestra investigación muestreamos tres puntos localizados dentro del área del puente, para su análisis en el laboratorio, realizando un total de tres monitoreos en el periodo comprendido del mes de Junio al mes de Agosto del año 2017. Los resultados obtenidos mediante procedimientos instrumentales de laboratorio indicaron valores ligeramente por encima del límite máximo permitido por el TULSMA en algunos de los parámetros analizados, como la dureza, sólidos disueltos totales, conductividad, cloruros, sulfato, hierro, Coliformes totales, mientras que, para el $\mathrm{pH}$, color, sabor y manganeso y otros, los valores estuvieron dentro de los estándares establecidos por esta norma. Y por lo tanto se concluye que las aguas del Estero Salado del sector analizadas no están gravemente contaminadas y por lo tanto son de aceptable calidad para la flora y fauna. Finalmente se determinó que el punto $\mathrm{N}^{\circ} 3$ correspondiente al extremo del puente es el que presentan ligera contaminación. Se recomienda a las autoridades competentes establecer y ejecutar campañas de saneamientos básicos, planes de monitoreos y remediación.

Palabras clave: Contaminación físico-química y microbiológica, agua del Estero Salado, puente Gómez Rendón, TULSMA, cantón Guayaquil. 


\begin{abstract}
In this research we did of a concrete study to evaluate the levels of contamination of the waters of the Estero Salado located in the Gómez Rendón bridge, Guayaquil province, Guayas province, whose objective was to determine the physico-chemical and microbiological contamination of waters of the Estero Salado, South-West sector of the city of Guayaquil-Ecuador, using instrumental procedures of laboratories undertaking actions of education and environmental prevention. In order to carry out our research, we sampled three points located within this area of the bridge, for analysis in the laboratory, performing a total of three monitoring in the period from June to August of 2017. The results obtained through In addition, the use of TULSMA as a method for the determination of the $\mathrm{pH}$ of the samples was performed in the same way as the $\mathrm{pH}$, color, flavor and manganese and others, the values were within the standards established by this standard. And therefore it is concluded that the waters of the estuarine estuary of the sector analyzed are not seriously contaminated and therefore are of acceptable quality for the flora and fauna. Finally, it was determined that point No. 3 corresponding to the end of the bridge is the one with slight contamination. It is recommended that the competent authorities establish and carry out basic sanitation campaigns, monitoring and remediation plans.
\end{abstract}

Key words: Physico-chemical and microbiological contamination, salt marshes water, Gómez Rendón bridge, TULSMA, Guayaquil canton. 


\section{Introducción.}

En la ciudad, el $70 \%$ de las industrias registradas se encuentran en la zona cercana a los esteros, de ellas, la industria de productos alimenticios representa el $70 \%$ del número total de plantas industriales, se estima un vertimiento anual de 600`000,000 de metros cúbicos, con un total de las descargas, la Industria de aceite y grasa aportan 6’000,000 de metros cúbicos por años, especialmente en Guayaquil, y la industria metal mecánica contribuye con un vertimiento de 16’000,000 de metros cúbicos por año (Vargas Cruz, 2015).

En Guayaquil, hay varias zonas donde se ubican las industrias en las riberas de los esteros, sur y muy en especial en la zona suburbana como el sector del puente de Gómez Rendón, los vertimientos industriales y domésticos van directo a estas zonas estearinas (CEVALLOS WONG, 2015)

El gobierno nacional, a través del Ministerio del Ambiente, y la Municipalidad de Guayaquil consideran como una de sus principales aspiraciones ambientales, mejorar la calidad de las aguas del Estero Salado, que se han venido deteriorando en razón de las descargas contaminantes que recibe, tanto de la industria como de los asentamientos humanos que año a año incrementan el área poblada de la ciudad, debido a las descargas de aguas negras domésticas e industriales al estero Salado sector Sur-Oeste, se ha ocasionado en las zonas aledañas y en el cuerpo de agua una alarmante contaminación con graves repercusiones caracterizado por el mal olor de sus aguas, un color negruzco y falta de oxígeno disuelto, todo lo cual repercute de manera especial sobre la existencia de vida superior en sus aguas (WOLF, 2003).

El Estero Salado fue por décadas ícono natural y termómetro ambiental de la ciudad de Guayaquil, con una vegetación compuesta por el $90 \%$ de mangle, que aporta a regular el clima de la 
urbe porteña, y constituyó un paraíso para las conchas, cangrejos, mejillones y una variedad de peces; así como cerca de un centenar de aves que tienen su hábitat en el estero salado (Vargas Cruz, 2015)

Como se conoce este importante brazo de mar comienza en el canal de Morro y atraviesa la ciudad con $60 \mathrm{Km}$ de ramales, semejantes a las arterias del corazón, hoy por hoy ese corazón llamado Estero Salado está enfermo, tiene dificultades para respirar y requiere de una cirugía mayor urgente, esto significa que en el estero Salado sus aguas lucen un color negruzco y emiten un olor de alimentos podridos, especialmente en el tramo sur, donde el cuerpo hídrico está repleto de sedimentos sin oxígeno constituido de residuos domésticos e industriales (Valencia, 2001).

La ciudad de Guayaquil, la más poblada, económicamente activa e industrializada del país, debe a través de sus instituciones gubernamentales y municipales, emprender todo tipo de acciones para lograr la remediación ambiental de este tramo del Estero Salado, que debe ser fundamentado en mejorar la calidad del agua, identificando los niveles de contaminación presentes en dicho ecosistema identificando las principales fuentes de contaminación del Estero Salado como son las aguas servidas domésticas, las industriales, los desecho sólidos vertidos por la población, las aguas lluvias y las aguas negras que entran por vía directa ubicando las zonas de estudio en sitios estratégicos y tratar el problema realizando varios proyectos de investigación debidamente financiados y sustentables que permitan la recuperación y mitigación del impacto ambiental planteado.

En el presente trabajo de investigación se van a determinar los niveles de contaminación físico-químicos y microbiológicos de las aguas del Estero Salado, en el sector del puente de Gómez Rendón, de la ciudad de Guayaquil, en el año 2017. 


\section{Metodología.}

Nuestra área de estudio, será en el estero salado sector puente Gómez Rendón, de la ciudad de Guayaquil, en dicha zona del puente, se lo ha dividido en tres puntos que a continuación se detallan:

1.- PUNTO UNO: Se localiza en la parte de la orilla de la avenida Barcelona.

2.- PUNTO DOS: Se localiza en la parte central del puente Gómez Rendón.

3.- PUNTO TRES: Se localiza en la parte extrema de la orilla de la avenida Barcelona.

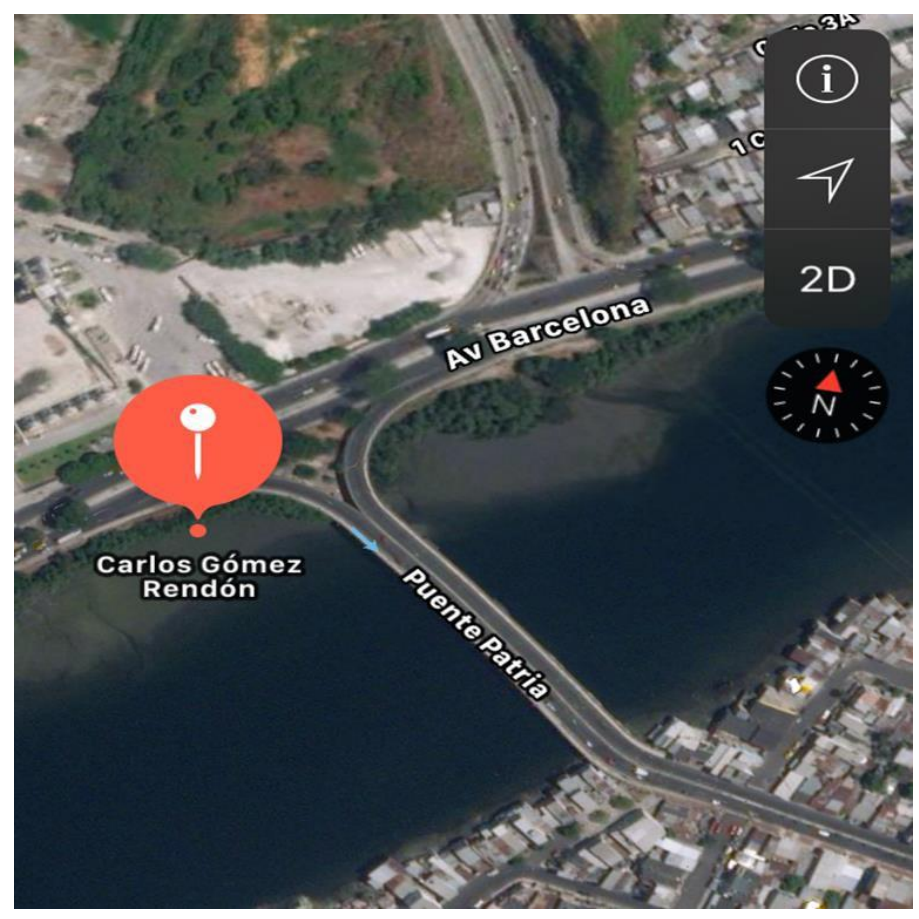

Grafica $N^{\circ} 1$ Zona de investigación

Periodo de ejecución: El primer muestreo se ejecutará en los días posteriores de ser aprobado el presente proyecto entre los meses de Junio - Julio - Agosto del 2017; se muestreará en pleamar, acontinuación, se detallan las fechas de monitoreo: 


\section{Fechas de monitoreo}

\begin{tabular}{|c|c|c|}
\hline Monitoreo 1 & Monitoreo 2 & Monitoreo 3 \\
\hline 5 & $5 \mathrm{Jul}$ & $\begin{array}{cc}5 & \text { Agosto } \\
2017\end{array}$ \\
\hline
\end{tabular}

\section{Tipo y diseño de investigación}

\section{Tipo}

Es una investigación Descriptiva- explicativa, comparativa con un enfoque metodológico cuantitativo, que parte de una población infinita, que se basa en la recolección, ordenamiento y análisis de los datos procedentes de un determinado conjunto de observaciones, de parámetros físicos, químicos y microbiológicos, obtenidas del agua del Estero Salado al sur-Oeste de la ciudad de Guayaquil, para determinar el nivel de contaminación de este sector, como parte inicial de la investigación se hizo una amplia revisión bibliográfica para conocer los antecedentes, descripciones, estudio de la flora y fauna del sector; se hicieron visitas a dependencias gubernamentales y municipales y una revisión exhaustiva del marco legal vigente.

\section{Diseño}

El diseño de la investigación para determinar el nivel de contaminación físico química y microbiológica del agua y sedimento del Estero Salado sector Sur-Oeste de la ciudad de Guayaquil es de tipo descriptiva - explicativa con un enfoque metodológico cuantitativo, y de comparación simple, debido a que se debe primero planear un conjunto de pruebas experimentales de tal manera que los datos obtenidos en el laboratorio, puedan observarse, compararse, y analizarse para obtener conclusiones válidas y objetivas del proceso de investigación. 


\section{Población, muestra y muestreo}

\section{Población}

La población a la que tiene alcance la investigación estará constituida por las aguas del Estero Salado, sector sur-oeste de la ciudad de Guayaquil cuya ubicación donde se realizará el trabajo, es desde el Primer Punto hasta el Tercer Punto del puente de Gómez Rendón, por consiguiente, la investigación que se realizará, permitirá obtener la información básica de los parámetros físico-químicos y microbiológicos de las aguas, para determinar una apropiada evaluación sobre el nivel de contaminación de este sistema estuario.

\section{Muestra}

Se recogerán las muestras en el sector norte del Estero Salado de la ciudad de Guayaquil, el tamaño de las muestras y el tipo de muestreo es Probabilística, no aleatoria tomando en cuenta que las aguas del estero salado en la que se determinarán sus niveles de calidad se lo hará en tiempo de flujo de marea para obtener muestras de agua en orilla, centro y extremo en cada perfil del puente:

Punto uno: Es la zona superior del puente Gómez Rendón, en la que se obtuvo muestras de orilla, es considerado como el sector más contaminado, según estudios de calidad ambiental realizados por Lahmeyer Cimentaciones, tiene menor influencia de las mareas y es considerada como la zona más afectada por las industrias, aguas residuales, residuos sólidos, etc como el puente sirve como vía de transporte, se podrá observar mucha basura arrojada en el área y también una descarga de escorrentía, el nivel de la rivera ha sido afectado con construcciones de viviendas y desechos de materiales y desechos sólidos. 
Punto dos: Es la zona central del puente Gómez Rendón, en la que se obtuvo muestras en la misma forma del punto anterior, recibe aguas de escorrentías de las calles cercanas al puente, hay olores desagradables, desperdicios sólidos en dicho estero, basura flotando, en dicho sector hay mayor densidad de mangles y garzas

Punto tres: Es la zona final del Puente Gómez Rendón, en la que se obtuvo muestras en la misma forma que los puntos anteriores, hay fácil acceso a la orilla opuesta a la avenida Barcelona.

\section{Muestreo}

La zona Sur-Oeste del Estero Salado de la ciudad de Guayaquil para objeto del muestreo será dividido en 3 puntos referenciados con GPS (Global Positionning System), modelo Garmn Etrex, para determinar la localización exacta (coordenadas) del área de investigación y en cada estación se medirán in situ y en el laboratorio (ex situ), los parámetros de la calidad del agua.

\begin{tabular}{|c|c|c|c|}
\hline Indicador & $\begin{array}{c}\text { Lugar de } \\
\text { medición }\end{array}$ & Método a utilizar & $\begin{array}{c}\text { Unidades } \\
\text { de medida }\end{array}$ \\
\hline Temperatura & In situ & Potenciómetro Mettler Toledo & o \\
\hline $\begin{array}{c}\text { pH } \\
\text { Sólidos totales } \\
\text { disueltos }\end{array}$ & In situ & $\begin{array}{c}\text { Potenciómetro Mettler Toledo } \\
\text { In situ } \\
\text { Standard Methods 2540B }\end{array}$ & $\mathrm{UpH}$ \\
\hline $\begin{array}{c}\text { Turbidez y } \\
\text { salinidad }\end{array}$ & In situ & Turbidímetro & $\mathrm{mT} / \mathrm{L}$ \\
\hline $\begin{array}{c}\text { Oxígeno } \\
\text { disuelto }\end{array}$ & In situ & $\begin{array}{c}\text { Instrumental THERMO } \\
\text { SCIENTIFIC(oxímetro) }\end{array}$ & $\mathrm{mg} / \mathrm{L}$ \\
\hline $\begin{array}{c}\text { Demanda } \\
\text { Bioquímica de } \\
\text { Oxígeno }\end{array}$ & Ex situ & HACH LBOD101 & $\mathrm{mg} / \mathrm{L}$ \\
\hline $\begin{array}{c}\text { Demanda } \\
\text { Química de } \\
\text { Oxígeno }\end{array}$ & Ex situ & Cary 60 & $\mathrm{mg} / \mathrm{L}$ \\
\hline Nitratos & Ex situ & Cary 60 & $\mathrm{mg} / \mathrm{L}$ \\
\hline Fosfatos & Ex situ & EPA 6020A & $\mathrm{mg} / \mathrm{L}$ \\
\hline
\end{tabular}




\begin{tabular}{|c|c|c|c|}
\hline $\begin{array}{c}\text { Coliformes } \\
\text { Fecales }\end{array}$ & Ex situ & $\begin{array}{c}\text { API-5.8-04-01-00M27 } \\
\text { (Standard Methods 21th } \\
\text { 9221ABCE)STANDARD } \\
\text { METHODS 21th 9221 ABCE }\end{array}$ & $\mathrm{NMP} / 100 \mathrm{~mL}$ \\
\hline $\begin{array}{c}\text { Coliformes } \\
\text { Totales }\end{array}$ & Ex situ & $\begin{array}{c}\text { API-5.8-04-01-00M22. } \\
\text { (Standard Methods 21th } \\
9221 \mathrm{ABC}) \\
\text { API-5.8-04-01-00M22. } \\
\text { (Standard Methods 21th } \\
\text { 9221ABC) } \\
\text { API-5.8-04-01-00M22.(Standard } \\
\text { Methods 21 th 9221 ABC) }\end{array}$ & $\mathrm{NMP} / 100 \mathrm{~mL}$ \\
\hline
\end{tabular}

Tabla $N^{o}$ 2: Tipos de métodos de análisis de aguas y unidades de medidas

\section{Material y métodos.}

Para la evaluación de las condiciones ambientales, y que constan en los términos de referencia para el Monitoreo Ambiental del dragado en cada estación de muestreo se midieron los niveles de oxígeno disuelto (OD), demanda bioquímica de oxígeno (DBO5), temperatura $\left({ }^{\circ} \mathrm{C}\right.$ ), salinidad, pH, Sólidos Disueltos Totales (TDS), turbidez, niveles de nitritos, nitratos y fosfatos.

\section{Resultados.}


TABLA N³: INDICADORES FÍSICO-QUÍMICOS DE LA CALIDAD DEL AGUA DEL ESTERO SALADO, UBICADOS EN EL PUENTE GOMÉZ RENDÓN, CANTÓN GUAYAQUIL, PROVINCIA DEL GUAYAS.

MES: JUNIO AÑO: 2017

FECHA: 05 JUNIO DE 2017

\section{ELABORADO POR: ALBERTO HERNÁNDEZ GAVILANES}

\begin{tabular}{|c|c|c|c|c|c|}
\hline $\begin{array}{c}\text { AREA DE } \\
\text { ESTUDIO }\end{array}$ & $\mathrm{pH}$ & Temperatura & $\begin{array}{c}\text { Solidos } \\
\text { disueltos } \\
\text { totales }\end{array}$ & $\begin{array}{c}\text { Conductividad - } \\
\text { salinidad }\end{array}$ & Turbidez \\
\hline $\begin{array}{c}\text { Unidades de } \\
\text { medida }\end{array}$ & $\mathbf{U p H}$ & ${ }^{\circ} \mathbf{C}$ & $\mathbf{m g} / \mathbf{L}$ & $\mu \mathbf{S} / \mathbf{c m}$ & NTU \\
\hline P1 & 7,36 & 24,2 & 28,9 & 561 & 171 \\
\hline P2 & 7,36 & 24,3 & 28,9 & 561 & 171,5 \\
\hline P3 & 7,35 & 24,4 & 28,91 & 563 & 171 \\
\hline Promedio & 7,35 & 24,3 & 28,9 & 561,66 & 171,16 \\
\hline $\begin{array}{c}\text { Desviación } \\
\text { estándar }\end{array}$ & 0,01 & 0,122 & 0,007 & 1,99 & 0,28 \\
\hline
\end{tabular}


Tabla N4: INDICADORES FÍSICO-QUÍMICOS DE LA CALIDAD DEL AGUA DEL ESTERO SALADO, UBICADOS EN EL PUENTE GOMÉZ RENDÓN, CANTÓN GUAYAQUIL, PROVINCIA DEL GUAYAS.

MES: JULIO AÑO: 2017

FECHA: 05 JULIO DE 2017

ELABORADO POR: ALBERTO HERNÁNDEZ GAVILANES

\begin{tabular}{|c|c|c|c|c|c|}
\hline $\begin{array}{c}\text { AREA DE } \\
\text { ESTUDIO }\end{array}$ & $\mathrm{pH}$ & Temperatura & $\begin{array}{c}\text { SOLIDOS } \\
\text { DISUELTOS } \\
\text { TOTALES }\end{array}$ & $\begin{array}{c}\text { CONDUCTIVIDAD } \\
- \text { SALINIDAD }\end{array}$ & TURBIDEZ \\
\hline $\begin{array}{c}\text { Unidades } \\
\text { de medida }\end{array}$ & UpH & ${ }^{\circ} \mathbf{C}$ & $\mathbf{m g} / \mathbf{L}$ & $\mu \mathbf{S} / \mathbf{c m}$ & NTU \\
\hline P1 & 7,33 & 22,5 & 29,3 & 560 & 169 \\
\hline P2 & 7,30 & 22,5 & 29,35 & 560 & 169 \\
\hline P3 & 7,31 & 22,6 & 29,4 & 560 & 169 \\
\hline Promedio & 7,31 & 22,5 & 29,35 & 560 & 169 \\
\hline $\begin{array}{c}\text { Desviación } \\
\text { estándar }\end{array}$ & 0,009 & 0,113 & 0,007 & 0 & 0 \\
\hline
\end{tabular}


Tabla N5: INDICADORES FÍSICO-QUÍMICOS DE LA CALIDAD DEL AGUA DEL ESTERO SALADO, UBICADOS EN EL PUENTE GOMÉZ RENDÓN, CANTÓN GUAYAQUIL, PROVINCIA DEL GUAYAS

MES: AGOSTO AÑO: 2017

FECHA: 05 AGOSTO DE 2017

ELABORADO POR: ALBERTO HERNÁNDEZ GAVILANES

\begin{tabular}{|c|c|c|c|c|c|}
\hline $\begin{array}{c}\text { AREA DE } \\
\text { ESTUDIO }\end{array}$ & $\mathbf{p H}$ & Temperatura & $\begin{array}{c}\text { SOLIDOS } \\
\text { DISUELTOS } \\
\text { TOTALES }\end{array}$ & $\begin{array}{c}\text { CONDUCTIVIDAD } \\
- \text { SALINIDAD }\end{array}$ & TURBIDEZ \\
\hline $\begin{array}{c}\text { Unidades } \\
\text { de medida }\end{array}$ & UpH & ${ }^{\circ} \mathbf{C}$ & $\mathbf{m g} / \mathbf{L}$ & $\boldsymbol{\mu S} / \mathbf{c m}$ & NTU \\
\hline P1 & 7,31 & 23 & 29,15 & 564 & 173 \\
\hline P2 & 7,32 & 23,1 & 29,2 & 564 & 173 \\
\hline P3 & 7,31 & 23,2 & 29,1 & 564,5 & 173 \\
\hline Promedio & 7,315 & 23,1 & 29,15 & 564,16 & 173 \\
\hline $\begin{array}{c}\text { Desviación } \\
\text { estándar }\end{array}$ & 0,009 & 0,115 & 0,007 & 1,98 & 0 \\
\hline
\end{tabular}


Tabla Nº: INDICADORES FÍSICO-QUÍMICOS DE LA CALIDAD DEL AGUA DEL ESTERo SALAdo, UbICADOS EN EL PUENTE GOMÉz RENDón, CANTón GUAYAQUIL, PROVINCIA DEL GUAYAS

MES: JUNIO AÑO: 2017

FECHA: 05 JUNIO DE 2017

ELABORADO POR: ALBERTO HERNÁNDEZ GAVILANES

\begin{tabular}{|c|c|c|c|c|c|}
\hline $\begin{array}{c}\text { AREA } \\
\text { ESTUDIO }\end{array}$ & $\begin{array}{c}\text { Oxígeno } \\
\text { Disuelto } \\
\mathbf{m g} / \mathbf{L}\end{array}$ & $\begin{array}{c}\mathbf{D B O}_{\mathbf{5}} \\
\mathbf{m g} / \mathbf{L}\end{array}$ & $\begin{array}{c}\text { DQO } \\
\mathbf{m g} / \mathbf{L}\end{array}$ & $\begin{array}{c}\text { Nitratos } \\
\mathbf{m g} / \mathbf{L}\end{array}$ & $\begin{array}{c}\text { Fosfatos } \\
\mathbf{m g} / \mathbf{L}\end{array}$ \\
\hline $\mathbf{P 1}$ & 0,80 & 17 & 153 & 0,034 & 0,40 \\
\hline $\mathbf{P 2}$ & 0,80 & 17 & 153 & 0,033 & 0,41 \\
\hline $\mathbf{P 3}$ & 0,81 & 17 & 153,5 & 0,032 & 0,42 \\
\hline PROMEDIO & 0,803 & 17 & 153,1 & 0,033 & 0,41 \\
\hline $\begin{array}{c}\text { DESVIACIÓN } \\
\text { ESTÁNDAR }\end{array}$ & 0,015 & 0 & 0,42 & 0,001 & 0,001 \\
\hline
\end{tabular}


Tabla N²: INDICADORES FÍSICO-QUÍMICOS DE LA CALIDAD DEL AGUA DEL ESTERo SALAdo, UbICAdos EN EL PUENTE GOMÉz RENDón, CANTón GUAYAQUIL, PROVINCIA DEL GUAYAS

MES: JULIO AÑO: 2017

FECHA: 05 JULIO DE 2017

ELABORADO POR: ALBERTO HERNÁNDEZ GAVILANES

\begin{tabular}{|c|c|c|c|c|c|}
\hline $\begin{array}{c}\text { AREA } \\
\text { ESTUDIO }\end{array}$ & $\begin{array}{c}\text { Oxígeno } \\
\text { Disuelto } \\
\mathbf{m g} / \mathbf{L}\end{array}$ & $\begin{array}{c}\mathbf{D B O}_{\mathbf{5}} \\
\mathbf{m g} / \mathbf{L}\end{array}$ & $\begin{array}{c}\mathbf{D Q O} \\
\mathbf{m g} / \mathbf{L}\end{array}$ & $\begin{array}{c}\text { Nitratos } \\
\mathbf{m g} / \mathbf{L}\end{array}$ & $\begin{array}{c}\text { Fosfatos } \\
\mathbf{m g} / \mathbf{L}\end{array}$ \\
\hline $\mathbf{P 1}$ & 0,82 & 17,5 & 154 & 0,035 & 0,44 \\
\hline $\mathbf{P 2}$ & 0,83 & 17,5 & 154 & 0,034 & 0,43 \\
\hline $\mathbf{P 3}$ & 0,82 & 17,5 & 154 & 0,033 & 0,45 \\
\hline PROMEDIO & 0,825 & 17,5 & 154 & 0,034 & 0,44 \\
\hline $\begin{array}{c}\text { DESVIACIÓN } \\
\text { ESTÁNDAR }\end{array}$ & 0,005 & 0 & 0 & 0,001 & 0,001 \\
\hline
\end{tabular}


Tabla No8: INDICADORES FÍSICO-QUÍMICOS DE LA CALIDAD DEL AGUA DEL ESTERo SAlado, ubicados EN EL PUENTE GOMÉz RENDón, CANTón GUAYAQUIL, PROVINCIA DEL GUAYAS.

MES: AGOSTO AÑO: 2017

FECHA: 05 AGOSTO DE 2017

ELABORADO POR: ALBERTO HERNÁNDEZ GAVILANES

\begin{tabular}{|c|c|c|c|c|c|}
\hline $\begin{array}{l}\text { AREA } \\
\text { ESTUDIO }\end{array}$ & $\begin{array}{c}\text { Oxígeno } \\
\text { Disuelto } \\
\mathbf{m g} / \mathbf{L}\end{array}$ & $\begin{array}{c}\mathbf{D B O}_{\mathbf{5}} \\
\mathbf{m g} / \mathbf{L}\end{array}$ & $\begin{array}{c}\text { DQO } \\
\mathbf{m g} / \mathbf{L}\end{array}$ & $\begin{array}{c}\text { Nitratos } \\
\mathbf{m g} / \mathbf{L}\end{array}$ & $\begin{array}{c}\text { Fosfatos } \\
\mathbf{m g} / \mathbf{L}\end{array}$ \\
\hline $\mathbf{P 1}$ & 0,83 & 16,8 & 155 & 0,032 & 0,41 \\
\hline $\mathbf{P 2}$ & 0,83 & 16,8 & 155 & 0,032 & 0,41 \\
\hline P3 & 0,83 & 16,8 & 155 & 0,032 & 0,41 \\
\hline PROMEDIO & 0,83 & 16,8 & 155 & 0,032 & 0,41 \\
\hline $\begin{array}{c}\text { DESVIACIÓN } \\
\text { ESTÁNDAR }\end{array}$ & 0 & 0 & 0 & 0 & 0 \\
\hline
\end{tabular}


Tabla N9: INDICADORES MICROBIOLÓGICOS DE LA CALIDAD DEL AGUA, ESTERO SALADO, UBICADOS EN EL PUENTE GOMÉz RENDÓN, CANTON GUAYAQUIL, PROVINCIA DEL GUAYAS

ELABORADO POR: ALBERTO HERNÁNDEZ GAVILANES

\begin{tabular}{|c|c|c|c|c|c|c|}
\hline & \multicolumn{2}{|c|}{ 05 JUNIO } & \multicolumn{2}{c|}{ 05 JULIO } & \multicolumn{2}{c|}{ 05 AGOSTO } \\
\hline $\begin{array}{c}\text { AREA } \\
\text { ESTUDIO }\end{array}$ & $\begin{array}{c}\text { COLIFORMES } \\
\text { TOTALES } \\
\text { (NMP/100 mL) }\end{array}$ & $\begin{array}{c}\text { COLIFORMES } \\
\text { FECALES } \\
\text { (NMP/100mL) }\end{array}$ & $\begin{array}{c}\text { COLIFORMES } \\
\text { TOTALES } \\
\text { (NMP/100 mL) }\end{array}$ & $\begin{array}{c}\text { COLIFORMES } \\
\text { FECALES } \\
\text { (NMP/100mL) }\end{array}$ & $\begin{array}{c}\text { COLIFORMES } \\
\text { TOTALES } \\
(\mathrm{NMP} / 100 \mathrm{~mL})\end{array}$ & $\begin{array}{c}\text { COLIFORMES } \\
\text { FECALES } \\
(\mathrm{NMP} / 100 \mathrm{~mL})\end{array}$ \\
\hline P1 & $2,1 \times 10^{6}$ & $1,1 \times 10^{6}$ & $2,3 \times 10^{6}$ & $1,4 \times 10^{6}$ & $2,35 \times 10^{6}$ & $1,3 \times 10^{6}$ \\
\hline P2 & $2,1 \times 10^{6}$ & $1,1 \times 10^{6}$ & $2,3 \times 10^{6}$ & $1,4 \times 10^{6}$ & $2,35 \times 10^{6}$ & $1,3 \times 10^{6}$ \\
\hline P3 & $2,1 \times 10^{6}$ & $1,1 \times 10^{6}$ & $2,3 \times 10^{6}$ & $1,4 \times 10^{6}$ & $2,35 \times 10^{6}$ & $1,3 \times 10^{6}$ \\
\hline PROMEDIO & $2,1 \times 10^{6}$ & $1,1 \times 10^{6}$ & $2,3 \times 10^{6}$ & $1,4 \times 10^{6}$ & $2,35 \times 10^{6}$ & $1,3 \times 10^{6}$ \\
\hline $\begin{array}{c}\text { DESVIACIÓN } \\
\text { ESTÁNDAR }\end{array}$ & 0 & 0 & 0 & 0 & & 0 \\
\hline
\end{tabular}

\section{Conclusiones}

Calidad del agua

Para determinar la calidad del agua se realizan análisis físico-químicos en campo tales como el pH, temperatura, salinidad, sólidos totales disueltos y oxígeno disuelto; mientras que en el laboratorio se determinarán la DBO5, DQO, turbidez, nitratos, fosfatos, Coliformes totales, Coliformes fecales, 
Todos los parámetros realizados, tanto de calidad de agua se realizaron según el Standard Methods for the Examination of Water and Wastewater, 21 ${ }^{\text {a }}$ Edición, la EPA y el Soil Sampling and Methods of Analysis- Carter\&Lewis como lo establece el Texto Unificado de Legislación Ambiental Secundaria,

Respecto a los límites permisibles, para agua los resultados fueron comparados con el límite máximo permitido, de los resultados obtenidos se observa lo siguiente:

El potencial de hidrógeno $\mathrm{pH}$, es la concentración de los iones hidrógeno en el agua, un pH elevado indica una baja concentración de iones H+, y por tanto una alcalinización del medio, por lo contrario, un $\mathrm{pH}$ bajo indica la acidificación del medio, por la mayor concentración de iones hidrógeno, el $\mathrm{pH}$ en el agua fluctúa en un ciclo diurno, directamente influenciado por la concentración de $\mathrm{CO} 2$, por la densidad del fitoplancton, la alcalinidad total y la dureza del agua, estas variaciones tienen una repercusión muy importante sobre la vida acuática presente en el agua del Estero Salado, los valores determinados durante el análisis de las muestras de agua fueron 7,31, 7,35 y 7,315 unidades de $\mathrm{pH}$, los valores reportados, se encuentran dentro del rango establecido en la norma para la preservación de la flora y fauna en el medio acuático (6,5 a 9,5) (TULSMA).

La temperatura influye entre otras cosas; en la solubilidad de las sales presentes en el agua, los gases y en el pH, Los cambios de temperatura afectan directamente la tasa metabólica, pues se registra una mayor tasa metabólica cuando se incrementa la temperatura, lo que provoca un mayor consumo de oxígeno, los valores medidos en el sitio son $231{ }^{\circ} \mathrm{C}, 22,5^{\circ} \mathrm{C}, 24,3{ }^{\circ} \mathrm{C}$, los valores se encuentran dentro de los límites permisibles que establece el TULSMA, que tiene relación con la preservación de la flora y fauna. 
La salinidad es el contenido de sales disueltas en el agua y se expresan en $\square \mathrm{S} / \mathrm{cm}$.

En el Estero salado, encontramos valores altos y bajos de salinidad; ya que esto depende de la intrusión del agua marina durante los períodos del flujo y reflujo de la marea y de agua dulce producto de las escorrentías generadas durante la época de lluvias, los valores de salinidad estadísticamente son altos, este parámetro no se encuentra normado dentro de la Legislación ecuatoriana, la salinidad fue medida en el laboratorio y para la bocatoma de entrada fue de 564,16 $\square \mathrm{S} / \mathrm{cm}, 560 \square \mathrm{S} / \mathrm{cm}, 561,66 \square \mathrm{S} / \mathrm{cm}$

La turbidez de un agua superficial se debe principalmente a la presencia de los sólidos en suspensión (microorganismos, arcillas, limos, fitoplancton, etc) los valores encontrados son: 173 NTU, 169 NTU, 171,16 NTU, La diferencia entre los valores mencionados se debe fundamentalmente al movimiento del agua, el día de la visita la marea estaba en reflujo,

Oxígeno disuelto $(\mathrm{O} 2)$, es el oxígeno libre que se encuentra en el agua, elemento vital para la supervivencia de todas las formas de vida acuática, La solubilidad del oxígeno depende de la concentración de sales disueltas y sobre todo de la temperatura y presión atmosférica, el grado de saturación del oxígeno está directamente influenciado por el $\mathrm{pH}$ y la temperatura, pero su relación con la altitud es inversamente proporcional, como información de referencia, los valores de oxígeno disuelto determinados en el puente de Gómez Rendón fueron, 0,803 mg/l, 0,825 mg/l, 0,83 mg/l, según el TULSMA el límite máximo permisible para aguas marinas y estearinas en cuanto a concentración de oxígeno disuelto se refiere, no debe ser menor a 5 mg O2/l.

La demanda bioquímica de oxígeno, DBO5, es la cantidad de oxígeno disuelto requerido por los microorganismos aerobios para la degradación de la materia orgánica, se la emplea para 
determinar el grado de contaminación de un cuerpo de agua, y se mide después que han transcurrido cinco días de reacción, Los valores reportados por el laboratorio fueron de 17,0 mg O2/1, 17,5 mg O2/1, 16,8 mg O2/1, de los resultados obtenidos podemos observar que el agua de por sí mantiene una cierta concentración de materia orgánica (como DBO5) pero que no denota una contaminación representativa,

Un análisis bacteriológico pone de manifiesto la presencia de bacterias que alteran y modifican la aptitud del agua para un determinado uso, de ahí que se decidió por realizar un análisis bacteriológico que permita evidenciar la calidad del agua desde este punto de vista, los valores encontrados como número más probable por cada $100 \mathrm{ml}$ de muestra para Coliformes totales arrojan los siguientes resultados, 2,1×106; 2,3x106 y 2,35x106 NMP/100 ml respectivamente, en el caso de los Coliformes fecales arrojan los siguientes resultados 1,1x106; 1,4x106 y 1,3x106 NMP/100 ml.

\section{Bibliografía.}

CEVALLOS WONG, J. (2015). DETERMINACION DE LA CONTAMINACIÓN FISCO QUIMICA DEL AGUA EN SECTOR NORTE DE LA CIUDAD DE GUAYAQUIL ECUADOR.

Valencia, M. (2001). Contaminantes del Estero Salado. Tecnico, Guayaquil- Ecuador.

Vargas Cruz, E. (NOVIEMBRE de 2015). trabajo de investigación. EVALUACIÓN DE LA CONTAMINACIÓN FÍSICO-QUÍMICA Y MICROBIOLOGICO. GUAYAS, GUAYAQUIL, ECUADOR.

WOLF, E. (2003). Municipio de Guayaquil tiene que responder por los asentamientos ilegales en zona protegida, nunca hizo control alguno". 